\title{
ESTUDIO DEL SUBGÉNERO ZAPETALOPTILA GOROCHOV \& LLORENTE, 2001 Y DESCRIPCIÓN DE CUATRO NUEVAS ESPECIES (PETALOPTILA PANTEL, 1890, ORTHOPTERA, GRYLLIDAE)
}

\author{
P. Barranco*
}

\begin{abstract}
RESUMEN
Se estudian las especies conocidas del subgénero Zapetaloptila y se describen cuatro nuevas: Petaloptila (Zapetaloptila) mogon sp. nov. de Villacarrillo (Jaén), P. (Z.) baenai sp. nov. de Córdoba y Jaén, $P$. (Z.) carabajali sp. nov. de la Sierra de Cazorla (Jaén) y $P$. (Z.) llorenteae sp. nov. de Rivas Vaciamadrid (Madrid). Se utiliza por primera vez la genitalia femenina como carácter diferenciador de especies para este sexo. Se redescribe $P$. (Z.) bolivari (Cazurro, 1888), se describe la hembra de $P$. (Z.) venosa Gorochov y Llorente, 2001, y la genitalia femenina de $P$. (Z.) barrancoi Gorochov y Llorente, 2001. Por último se incluyen unas claves dicotómicas para la determinación de las especies del subgénero.
\end{abstract}

Palabras clave: subgénero Zapetaloptila, Orthoptera, Gryllidae, especies nuevas, genitalia femenina. Petaloptila mogon sp. nov., P. baenai sp. nov., P. carabajali sp. nov., $P$. llorenteae sp. nov., P. (Z.) bolivari, P. (Z.) venosa, P. (Z.) barrancoi.

\begin{abstract}
Study of the subgenus Zapetaloptila Gorochov \& Llorente, 2001 and description of four new species (Petaloptila Pantel, 1890, Orthoptera, Gryllidae)

The known species of Spanish subgenus Zapetaloptila are studied and four new species are described: Petaloptila (Zapetaloptila) mogon sp. nov. from Villacarrillo (Jaen), $P$. (Z.) baenai sp. nov. from Cordoba and Jaen, P. (Z.) carabajali sp. nov. from Sierra de Cazorla (Jaen) and $P$. (Z.) llorenteae sp. nov. from Rivas Vaciamadrid (Madrid). It is the first time that genitalia is used to distinguish the females and it is described for each species. $P$. (Z.) bolivari (Cazurro, 1888) is redescribed as well as the female of $P$. (Z.) venosa Gorochov y Llorente, 2001. A key for specific determination of the subgenus is provided.

Key words: subgenus Zapetaloptila, Orthoptera, Gryllidae, new species, female genitalia. Petaloptila mogon sp. nov., P. baenai sp. nov., P. carabajali sp. nov., P. llorenteae sp. nov., P. (Z.) bolivari, P. (Z.) venosa, P. (Z.) barrancoi.
\end{abstract}

* Dpto. Biología Aplicada. Cite II-B. Universidad de Almería. 04120 Almería. España. 


\section{Introducción}

El género Petaloptila ha estado integrado por cuatro especies durante más de cincuenta años, una en Italia y las otras tres en la Península Ibérica. No obstante la distribución ibérica del género es muy amplia, existiendo en las colecciones de especialistas y del M.N.C.N. de Madrid numeroso material de procedencia diversa. El estudio de la genitalia masculina de las especies ha aportado una herramienta eficaz para la determinación y caracterización específica, lo que ha supuesto un incremento notable en el número de taxones conocidos, alcanzándose en la actualidad nueve especies agrupadas en tres subgéneros gracias a los trabajos de Olmo-Vidal y Hernando (2000) y Gorochov y Llorente (2001).

La adquisición de nuevo material procedente de cavidades del sur de España y la revisión de la colección del M.N.C.N. de Madrid, nos ha permitido describir cuatro nuevas especies y esclarecer un poco más los caracteres diferenciadores con las otras especies conocidas.

No obstante, tanto los dos trabajos mencionados, como el presente estudio, ponen de manifiesto la semejanza externa entre los diferentes taxones, cuya separación es únicamente fiable mediante el estudio de la genitalia. Si bien se profundiza en el estudio de otras estructuras como las tegminas en los machos y los epiproctos en ambos sexos.

La diferenciación de las hembras de las diferentes especies del género es complicada, tanto para el subgénero Petaloptila como para Zapetaloptila. Pues aún cuando se aprecian diferencias en la anchura y escotadura del borde posterior de la placa subgenital, forma del epiprocto y longitud del oviscapto, salvo para alguna especie que son caracteres válidos, en la mayoría de los casos las variaciones son sutiles y la propia variación intraespecífica hace muy difícil la validación de estos caracteres. Ya indicaba el propio Pantel (1890) la escasa validez de la placa subgenital para la separación de las hembras. Así, se ha llegado a señalar que la identificación de las hembras es muy difícil sin el estudio conjunto de machos asociados (Olmo-Vidal y Hernando, 2000).

Todo ello ha hecho necesario buscar un carácter fiable que identifique a las hembras de forma precisa. Este no ha sido otro que la genitalia femenina. Hubbell y Norton (1978) ya establecieron diferencias en la forma de la espermateca entre géneros próximos de grillos cavernícolas, si bien no indican diferencias a nivel específico. El estudio de la genitalia femenina presenta dificultades, pues en gene- ral, posee pocas piezas esclerotizadas y su ubicación es bastante interna (Fig. 1). Aunque la forma de la papila copulatriz, en cuyo centro desemboca la espermateca, posee formas características para cada especie y ya ha sido empleado por otros autores para la descripción de especies de grillos cavernícolas (Mesa et al., 1996). En el caso del género que nos ocupa los escleritos son muy visibles. La única dificultad que entraña el estudio de la genitalia femenina es que el ejemplar ha de estar conservado en alcohol u otro conservante, ya que la papila copulatriz se inserta muy profundamente y su extracción es relativamente sencilla y no acarrea la lesión de otras estructuras en los ejemplares conservados en estas condiciones.

Para la descripción de las tibias posteriores se ha seguido el criterio Otte y Alexander (1983); para la genitalia masculina se ha seguido la nomenclatura establecida por Gorochov y Llorente (2001) y para la femenina la adoptada por Alexander y Otte (1967).

\section{Descripciones taxonómicas}

Petaloptila (Zapetaloptila) mogon sp. nov. (Figs. 2A; 2F; 3A; 4A-C; 5A; 6A-B)

Material estudiado: Holotipo: $1 \sigma^{\top}$, Cueva de la Morciguilla, Villacarrillo, Jaén, 31-V-2002, (GEV leg.). Paratipos: $2 \sigma^{7} \sigma^{7}$, 1 ㅇ , mismos datos del holotipo; $10^{7}, 20$-IV-2002; 1 오, 24-III2002; 1 ㅇ , 31-V-2002; 1 오, Sima de la Fractura, Hornos, Jaén, 17-III-2002, (GEV leg.); 1 \& , Sima del Campamento, Hornos, Jaén, 2-III-2003, (GEV leg.). Tres paratipos, 2 우 우 y $10^{7}$ depositados en M.N.C.N. de Madrid, Cat. Tipos No 9456; el resto en la colección particular del autor.

DESCRIPCIÓN DEL MACHO: es la especie de mayor tamaño del género. Coloración con fondo claro, aunque el vertex, pronoto, dorso del abdomen y fémures posteriores salpicados de manchas pardo oscuras. Fastigio y frente castaños, vertex pálido. Bordes anterior y posterior del pronoto provistos de numerosas setas negras, faltando en el centro del posterior. Tegminas rojizas con el borde interno oscurecido, grandes, no llegan a alcanzar la mitad del tercer terguito abdominal y con el ápice prolongado y estrecho muy parecido a $P$. barrancoi, si bien se aprecian tres tenues venas longitudinales (Fig. 2A).

Glándula dorsal débilmente desarrollada, característica del subgénero Zapetaloptila (Fig. 2F). Epiprocto transverso con una depresión anterocentral subcircular y con sendas escotaduras laterales bien marcadas (Fig. 3A). Placa subgenital característica del género. Tibias posteriores armadas con 4 


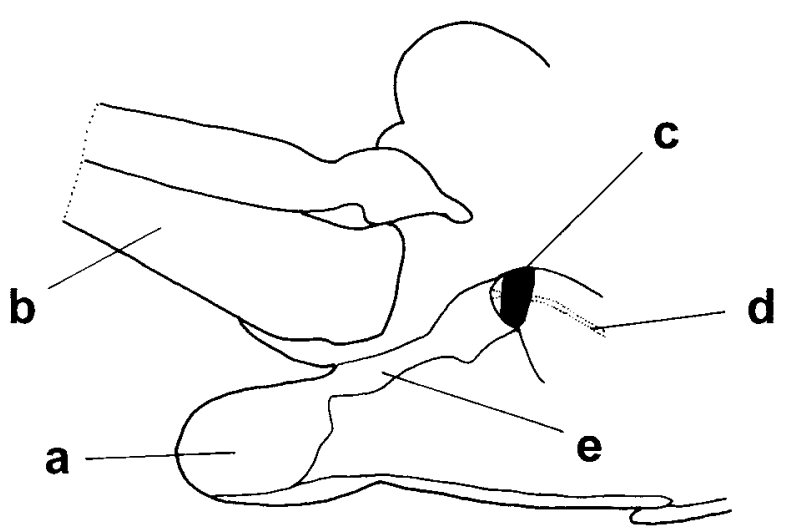

Fig. 1.- Localización y sección sagital de la genitalia femenina en Petaloptila sp. Abreviaturas: a) placa subgenital, b) oviscapto, c) papila copulatriz y esclerito, d) conducto de la espermateca, e) conducto genital.

Fig. 1.- Location and sagittal section of female genitalia of Petaloptila sp. Abreviatons: a) subgenital plate, b) ovipositor, c) copulatrix papilla and sclerite, d) spermathecal duct, e) genital chamber.

espolones 6-9 espinas externas y 4 espolones y 710 espinas internas.

Lóbulos pilosos del ápice del epifalo largos, cubriendo parcialmente éste. Epifalo desarticulado de los rami y escotado como en $P$. barrancoi, aunque el borde distal es más sinuoso y se aprecia un lóbulo triangular central prominente (Fig. 4A). Ectoparámeros con la proyección distal muy desarrollada, a modo de concha bivalva con el ápice lobulado no ramificado (Fig. 4B-C), con los bordes superior e inferior convexos; y la proyección proximal corta y un poco ensanchada en el ápice y curvada hacia arriba. Moldura de la placa de sujeción del espermatóforo ancha (como en $P$. venosa), escotada y con un pequeño diente romo dorsal en el centro. Apodemas endoparamerales anchos, cruzados con los rami en el ápice. Varilla guía prolongada hacia atrás hasta conectar con el endoparámero.

DESCRIPCIÓN DE LA HEMBRA: aspecto general como el del macho, de mayor tamaño, mucho más pigmentada. Dorso del vertex, pronoto y segmentos abdominales fuertemente pigmentados de color castaño compuesto por manchas irregulares y puntos de tamaño variable, interrumpido por una línea media dorsal clara a lo largo de todo el cuerpo. Dorso de los fémures posteriores manchados de oscuro. Pleuras y zona ventral del cuerpo claras, a excepción de la placa subgenital que es también oscura, al menos, en su mitad anterior. Tegmina típica del género, reducida a un pequeño lóbulo oval casi cubierto por el margen posterior del pronoto. Oviscapto curvado hacia abajo, de mayor longitud que el fémur posterior. Epiprocto transverso con una depresión central en el tercio anterior en punta de flecha y escotaduras laterales que provocan una débil depresión lateral de la porción distal (Fig. 5A). Genitalia femenina con el esclerito de la papila copulatriz en forma de herradura, estrecho, bastante aplanado y con una escotadura en el margen interno de cada una de los brazos que le dan una apariencia de punta de arpón al extremo, en algunos ejemplares el margen anterior presenta una pequeña escotadura central inferior (Figs. 6A-B).

MEDIDAS (en milímetros): longitud total: holotipo 20,2, O' 18-20,5, 우 21,7-23,5; longitud pronoto: holotipo 3, $\sigma^{\top} 3$, o 3-3,3; longitud de la tegmina: holotipo 5,9, ठ 5,8; longitud del fémur posterior: holotipo 14,2, ơ 12,9-13,3, ㅇ 12,9-14,4; longitud de la tibia posterior: holotipo 12,1, ơ 11,1-11,4, ㅇ 11,1-12,4; longitud del oviscapto: 14,4-16,2.

DisTRIBUCIÓN: por ahora sólo en cuevas de la provincia de Jaén.

DERIVATIO NOMINIS: nombre por aposición. Dedicamos esta especie a la localidad de Mogón, pedanía de Villacarrillo (Jaén) y antiguo asentamiento fenicio en cuyas proximidades se localiza la cueva donde se ha capturado el material que ha posibilitado la descripción de esta nueva especie.

\section{Petaloptila (Zapetaloptila) baenai sp. nov.} (Figs. 2B; 2G; 3B; 4D-F; 5F; 6K-L)

Material estudiado: Holotipo: $1 \sigma^{7}$, Cueva de la Negra, Rute, Córdoba, 9-II-2003, (A. Moreno y M. Baena leg.). Paratipos: $10^{7}$, mismos datos; 10", 19-I-2003, (A. Moreno leg.); $20^{7} \sigma^{7}$ La Casería de Cristóbal, Pte. de la Sierra, km 1,8, Jaén, 20-XI2003, (A. Castro Tovar leg.); 1 ㅇ, mismos datos, 31-I-2004. Un paratipo depositado en el M.N.C.N. de Madrid, Cat. Tipos $\mathrm{N}^{\circ}$ 9457; el resto en la colección del autor.

DESCRIPCIÓN DEL MACHO: coloración general muy pálida, cuerpo cubierto de fina pilosidad aterciopelada, también pálida. Cabeza ocrácea con seis líneas longitudinales dorsales, las dos centrales interrumpidas. Pronoto pálido con algunas manchas pardo claras; borde posterior de los paranotos marcados con una mancha oscura; margen anterior y posterior provistos de densas setas negras, más largas en el primero. Patas totalmente pálidas, salvo dos manchas pardo claras en la mitad distal 


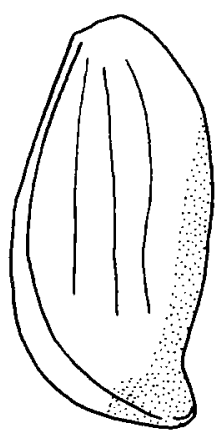

A

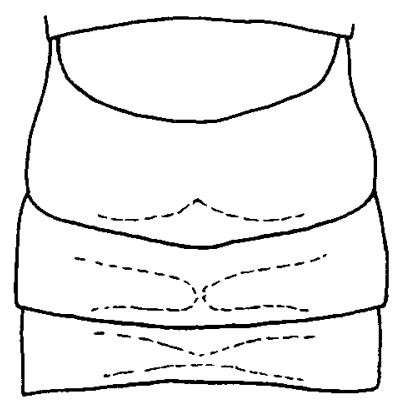

$\mathbf{F}$

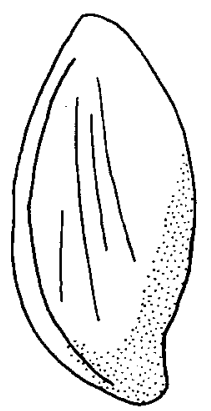

B

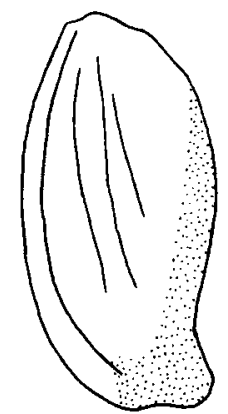

C

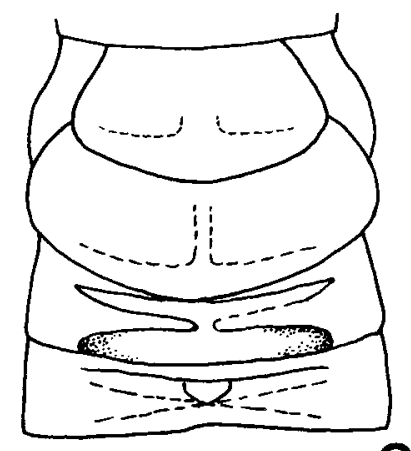

G
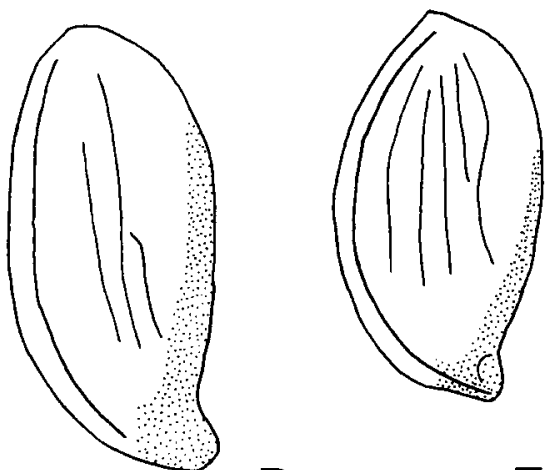

D

$\mathbf{E}$

Fig. 2.- Tegminas masculinas y glándulas dorsales: A, F) P. mogon sp. n.; B, G) P. baenai sp. n.; C) P. carabajali sp. n.; D) P. llorenteae sp. n.; E, H) P. bolivari.

Fig. 2.- Left male tegmen and dorsal protuberances: A, F) P. mogon sp. n.; B, G) P. baenai sp. n.; C) P. carabajali sp. n.; D) P. llorenteae sp. n.; E, H) P. bolivari.

de la cara interna del fémur posterior. Tegminas pardo rojizas con el borde interno y ápice oscurecidos con una franja negra; con cuatro tenues venas longitudinales y la vena lateral muy marcada en el margen externo; ápice escotado interiormente, típico del subgénero (Fig. 2B). Apenas alcanzan el borde anterior del tercer segmento abdominal.

Glándula dorsal algo más desarrollada que en otras especies del subgénero, consistente en un estrecho engrosamiento del margen posterior del segundo terguito abdominal y una pequeña protuberancia central en la mitad anterior del tercer terguito abdominal (Fig. 2G). Epiprocto subcuadrangular, con las escotaduras laterales poco marcadas y una pequeña depresión en forma de hacha en la mitad anterior (Fig. 3B). Placa subgenital característica del género. Tibias posteriores con 5 espolones superiores exter- nos y de 3 a 5 espinas, y 5 internos con 3 a 6 espinas (este número varía incluso en el mismo ejemplar).

Lóbulos pilosos del ápice del epifalo cortos. Epifalo ancho, articulado con los rami, profundamente escotado en el borde anterior, alcanzando la escotadura la mitad de la anchura de éste. Borde posterior con una invaginación a sendos lados del centro, de modo que éste es trilobulado (Fig. 4D). Ectoparámeros de aspecto bivalvo, proyección distal muy amplia, dilatada superiormente con el borde liso y convexo, borde inferior con una pequeña escotadura apical y convexo redondeado en el resto. Proyección proximal estrecha y prolongada en un tubérculo (Fig. 4E-F). Apodemas endoparamerales anchos y largos, superando la longitud de los rami. Endoparámero estrecho y con los cóndilos muy desarrollados. Moldura de la placa de sujeción del espermatóforo ancha, un poco deprimida en el 

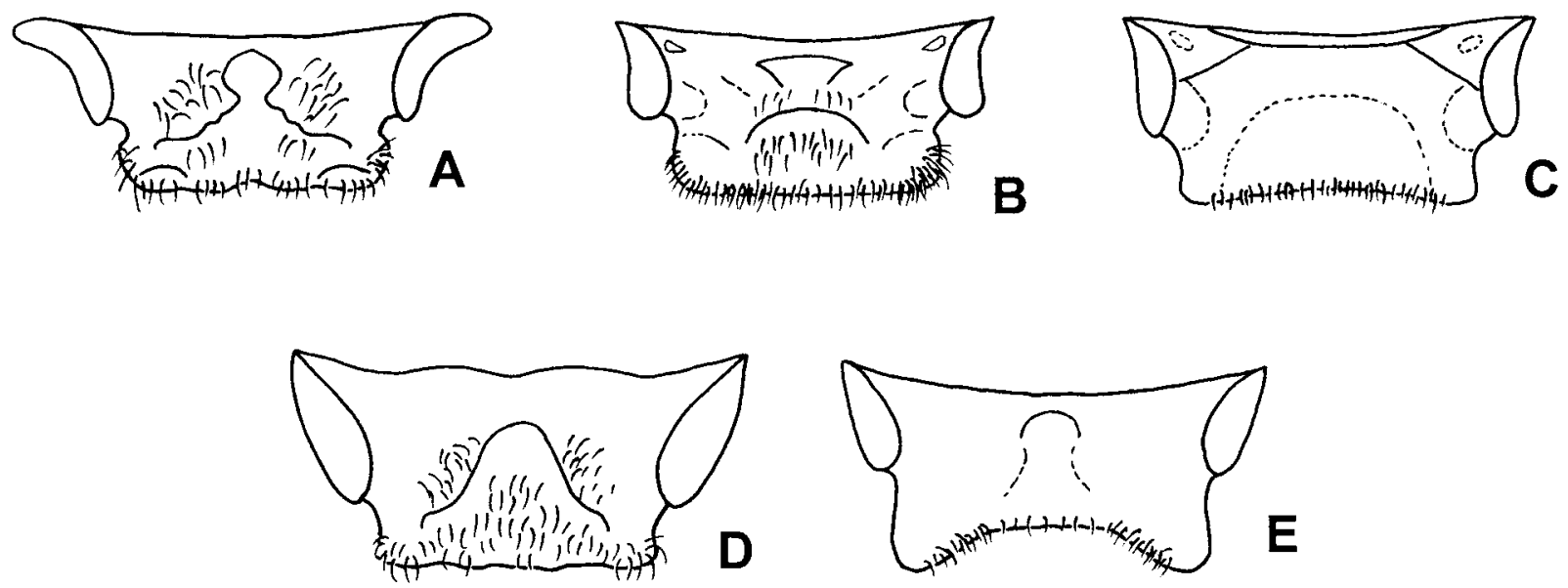

Fig. 3.- Epiprocto masculino: A) P. mogon sp. n.; B) P. baenai sp. n.; C) P. bolivari; D) P. barrancoi; E) P. venosa.

Fig. 3.- Male supra-anal plates: A) P. mogon sp. n.; B) P. baenai sp. n.; C) P. bolivari; D) P. barrancoi; E) P. venosa.

borde anterior, procesos laterales cortos, procesos espiniformes muy cortos y centro del borde posterior convexo. Varilla guía muy corta y ancha, no superando el arco del endoparámero.

DESCRIPCIÓN DE LA HEMBRA: aspecto general como el macho. Coloración muy pálida, con las manchas pardas muy poco destacadas, áreas pleurales y ventrales del cuerpo blancas. Líneas longitudinales de la cabeza muy poco patentes. Tegmina típica del género, reducida a un pequeño lóbulo oval casi cubierto por el margen posterior del pronoto. Oviscapto curvado hacia abajo, de mayor longitud que el fémur posterior. Epiprocto transverso (Fig. 5F), corto, semejante a $P$. carabajali sp. n., con el borde posterior recto, escotaduras laterales poco marcadas. Placa subgenital estrecha, con la escotadura apical netamente marcada.Genitalia femenina con el esclerito de la papila copulatriz en forma de herradura, ancho, bastante aplanado y estrechándose hacia los ápices, con los bordes laterales externos casi paralelos, lateralmente con aspecto casi rectangular (Figs. 6K-L).

MEDIDAs (en milímetros): longitud total: holotipo 14,7 , O' $14,9-16,8$, ㅇ 18,1; longitud pronoto: holotipo $2,3, \sigma^{\prime} 2,1-2,8$, ㅇ 2,8; longitud de la tegmina: holotipo 4,4, $0^{\pi} 4,0-4,4$; anchura de la tegmina: holotipo 2,2, ơ 1,9-2,4; longitud del fémur posterior: holotipo $10,3, \sigma^{7} 8,1-11,2$, ㅇ 11,4; longitud de la tibia posterior: holotipo 8,3, $\sigma^{\pi} 6,3-9,3$, o 9,2; longitud del oviscapto: 12,5 .
DISTRIBUCIÓN: en cuevas de la provincia de Córdoba y epigeo en Jaén. Los ejemplares de Jaén son de menor tamaño que los de Córdoba.

DERIVATIO NOMINIS: dedicamos esta especie al hemipterólogo D. Manuel Baena Ruiz que nos ha confiado el material para su estudio.

COMENTARIOS: Deben adscribirse a esta nueva especie las 4 ninfas citadas como $P$. bolivari de Cueva Morrón Grande, actualmente Cueva de la Negra, (Rute, Córdoba) en Barranco et al. (1996).

\section{Petaloptila (Zapetaloptila) carabajali sp. nov.} (Figs. 2C; 4G-I; 5B; 6C-D).

Material estudiado: Holotipo: $10^{x}$, Cueva del Sagreo, Pto. Las Palomas, Sierra de Cazorla, Jaén, 26-III-1991, (Carabajal leg.). Paratipos: $3 ㅇ ㅜ$ 으, mismos datos; 1 , , misma localidad, 2-V-1999; 3 우 ㅇ, Cueva Covarona, La Muela, Santiago de la Espada, Jaén, 9-XI-2002, (V. Ortuño y J.L. Lencina leg.). Dos paratipos depositados en el M.N.C.N. de Madrid, Cat. Tipos N $^{\circ}$ 9458; el resto en la colección del autor.

DESCRIPCIÓN DEL MACHO: Aspecto general pálido, concolor, sin apenas diferenciarse manchas o líneas más oscuras en el vertex, pronoto y tibias posteriores. Bordes anterior y posterior del pronoto casi sin setas, salvo algunas dispersas, cortas y negras en el anterior. Tegminas grandes, rojizas con el ápice y borde interno negros, alcanzando el margen posterior del tercer terguito abdominal, largas y estrechas, con la escotadura apical interna menos 

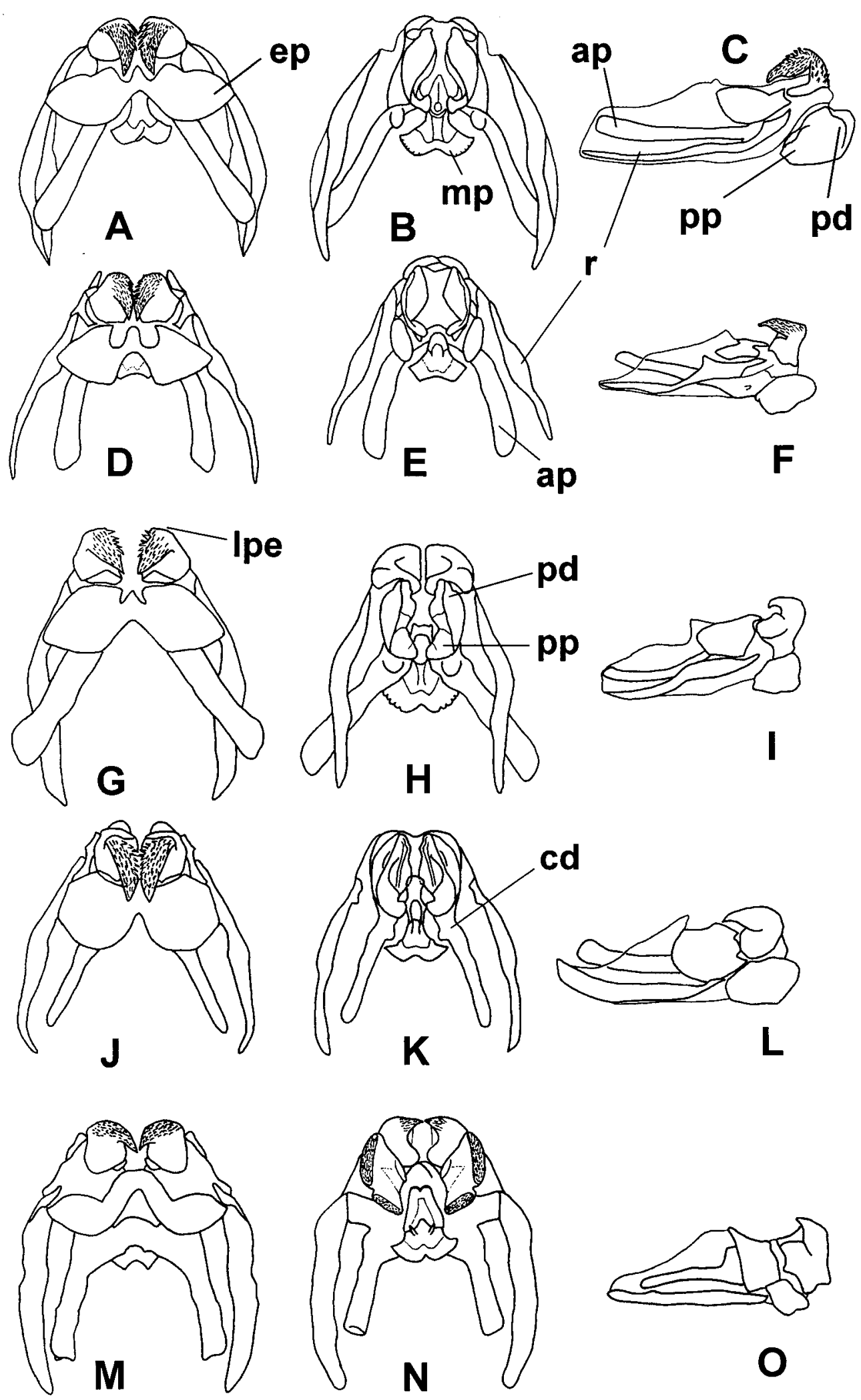
marcada y el lóbulo apical más corto; se aprecian tres venas además de la lateral, si bien únicamente la central es completa (Fig. 2C). Tibias posteriores con 5 espolones y 4 espinas externas y 4 espolones y 4 espinas internas.

Glándula dorsal muy poco desarrollada, como en $P$. mogon, sin ningún tipo de protuberancia. Epiprocto subcuadrangular, muy semejante al de $P$. baenai, aunque la depresión central del tercio anterior es más estrecha.

Lóbulos pilosos del ápice del epifalo cortos. Epifalo muy ancho, articulado lateralmente con los rami, borde anterior escotado, borde posterior casi continuo, interrumpido casi únicamente por dos estrechas incisiones convergentes en el medio que producen un pico central (Fig. 4G). Moldura de la placa de sujeción del espermatóforo muy ancha como en P. bolivari (Fig. 4N), borde anterior escotado, expansiones laterales anchas, borde posterior estrecho en el centro, formando una pequeña concavidad entre los procesos espiniformes. Ectoparámero de aspecto bivalvo, proyección distal no ramificada, muy amplia y convexa, con el borde superior casi recto y el inferior sinuoso con una amplia convexidad (Fig. 4H-I). Proyección proximal corta y roma. Apodemas endoparamerales anchos y robustos, cruzados con los rami y de la longitud de éstos. Endoparámero estrecho, poco visible, tapado por la moldura de la placa de sujeción del espermatóforo, cóndilos prominentes. Varilla guía corta y ancha.

DESCRIPCIÓN DE LA HEMBRA: cuerpo de coloración general pálida como en el macho. Terguitos meso, metatorácicos y abdominales más castaños, con una estrecha línea clara central que abarca todo el dorso desde el pronoto al extremo posterior del cuerpo. Tegminas muy pequeñas, ovales, horizontales, casi cubiertas por el margen posterior del pronoto. Epiprocto muy parecido al de $P$. bolivari y $P$. mogon, subtrapezoidal con el borde distal cóncavo, escotaduras laterales marcadas y depresión anterior central semicircular (Fig. 5B). Placa subgenital estrecha, con la escotadura apical poco profunda.
Papila copulatriz en visión dorsal con el esclerito ancho, abierto anteriormente y las ramas de éste divergentes (Fig. 6C). En visión lateral semejante a P. mogon pero algo más elevada (Fig. 6D).

MEdIDAs (en milímetros): longitud total: holotipo 14,8, ㅇ 15,7-18; longitud pronoto: holotipo 2,9, O 2,6-2,8; longitud de la tegmina: holotipo 5,4; longitud del fémur posterior: holotipo 11,2, ᄋ 11,212; longitud de la tibia posterior: holotipo 10,3, o 9,4-11,4; longitud del oviscapto: 11,6-13.

Distribución: cuevas de la Sierra de Cazorla (Jaén).

DERIVATIO NOMINIS: dedicamos esta especie a nuestro amigo D. Emilio Carabajal Márquez, especialista en carábidos y pseudoescorpiones por la cesión del material de esta nueva especie.

\section{Petaloptila (Zapetaloptila) llorenteae sp. nov.} (Figs. 2D; 4J-L)

MATERIAL estudiado: Holotipo: $10^{7}$, río Jarama, Vaciamadrid, 14-VI-1967, (E. Ortiz leg.), M.N.C.N. Cat. Tipos No 9459; Petaloptila (Zapetaloptila), V. Llorente det. 2000. Paratipo: 1 오, mismos datos. Ambos ejemplares pertenecen a la colección del M.N.C.N. de Madrid.

DESCRIPCIÓN DEL MACHO: Aspecto general pálido, con algunas manchas pardas apreciables en el vertex. Borde anterior y posterior del pronoto con profusas setas oscuras, más densas en el anterior. Tegminas pardo rojizas, estrechas, con el margen interno y ápice negros. Escotadura apical típica del subgénero. Vena marginal bien marcada aunque no alcanza el ápice, otras tres venas más o menos marcadas son visibles (Fig. 2D).

Glándula dorsal irreconocible por la inserción del alfiler entomológico, si bien se aprecia un reborde engrosado en el borde posterior del segundo terguito abdominal. Epiprocto deformado en el ejemplar disponible, si bien las escotaduras laterales aparecen poco marcadas.

Lóbulos pilosos del ápice del epifalo prolongados anteriormente, cubriendo parcialmente el epi-

Fig. 4.- Genitalia masculina, visión dorsal, ventral y lateral: A-C) P. mogon sp. n.; D-F) P. baenai sp. n.; G-I) P. carabajali sp. n.; J-L) P. llorenteae sp. n.; M-O) P. bolivari. Abreviaturas: ap) apodema endoparameral, cd) cóndilos del endoparámero, ep) epifalo, lep) lóbulos pilosos del epifalo, $\mathrm{mp}$ ) moldura de la placa de sujeción del espermatóforo, pd) proyección distal del ectoparámero, pp) proyección proximal del ectoparámero, r) ramus.

Fig. 4.- Male Genitalia, dorsal, ventral and lateral views: A-C) P. mogon sp. n.; D-F) P. baenai sp. n.; G-I) P. carabajali sp. n.; J-L) P. llorenteae sp. n.; M-O) P. bolivari. Abreviations: ap) endoparameral apodeme, cd) endoparal process, ep) epiphallus, lep) hairy lobes of apex of epiphallus, $\mathrm{mp}$ ) mold of attachment plate of spermatophore, $\mathrm{pd}$ ) distal process of ectoparamere, $\mathrm{pp}$ ) proximal process of ectoparamere, r) ramus. 

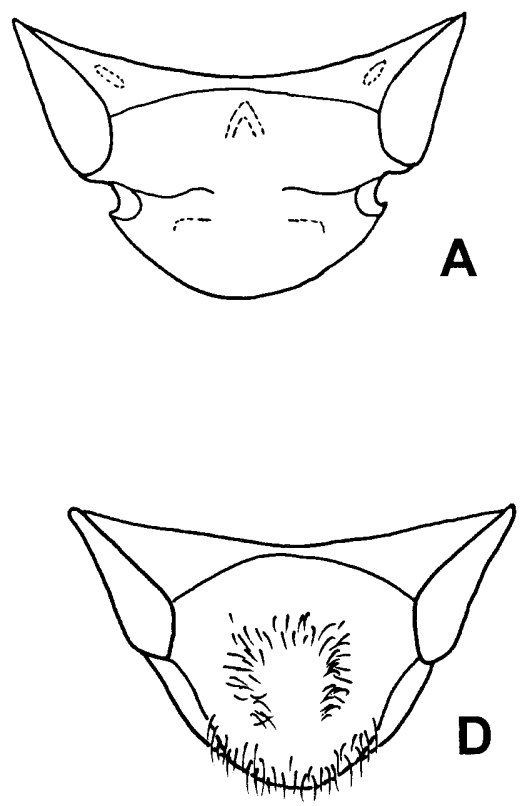
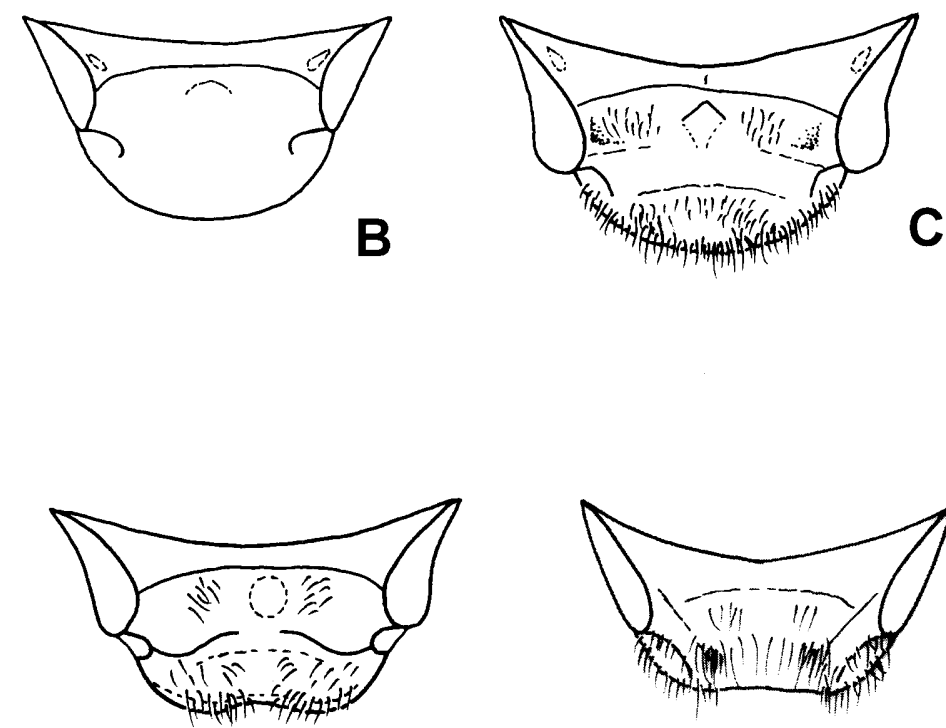

E

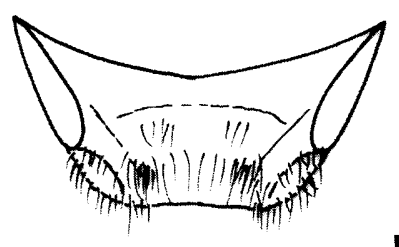

$\mathbf{F}$

Fig. 5.- Epiprocto femenino: A) P. mogon sp. n.; B) P. carabajali sp. n.; C) P. bolivari; D) P. barrancoi; E) P. venosa, F) P. baenai sp. n.

Fig. 5.- Female supra-anal plates: A) P. mogon sp. n.; B) P. carabajali sp. n.; C) P. bolivari; D) P. barrancoi; E) P. venosa, F) $P$. baenai $\mathrm{sp} . \mathrm{n}$.

procto. Epifalo muy ancho, ampliamente articulado lateralmente con los rami, borde anterior escotado centralmente, borde posterior casi recto, levemente deprimido en el medio con una pequeña protuberancia central aguda como en $P$. barrancoi (Fig. $4 \mathrm{~J})$. Ectoparámero de aspecto bivalvo, proyección distal no ramificada, muy dilatada y convexa, muy semejante a P. carabajali, aunque es más ancha y la convexidad del borde inferior es mayor y más interna. Proyección proximal corta y roma (Fig. 4K-L). Apodemas endoparamerales poco desarrollados, estrechos y relativamente cortos, no alcanzando la longitud de los rami. Endoparámero con los cóndilos medianamente desarrollados. Moldura de la placa de sujeción del espermatóforo ancha y un poco escotada anteriormente, con expansiones laterales estrechas, borde posterior estrecho con los procesos espiniformes bastante juntos. Varilla guía ancha y larga, dilatada en el ápice.

DESCRIPCIÓN DE LA HEMBRA: el único ejemplar disponible está muy deteriorado, carente de bastantes patas y con el oviscapto incompleto y con algunas estructuras deformadas o contraídas por la conservación en seco. Aspecto general como el del macho. Terguitos abdominales castaños, salvo en el margen posterior que poseen una estrecha franja transversa clara. Paranotos más claros que el disco. Tegminas típicas del género, reducidas, ovales, casi cubiertas por el margen posterior del pronoto. Epiprocto grande, prolongado posteriormente, márgenes laterales expandidos como en $P$. barrancoi (Fig. 5D), escotaduras laterales muy marcadas. Depresión anterocentral en forma de U invertida.

MEdIDAs (en milímetros): longitud total: holotipo 12,4 , 13,6; longitud pronoto: holotipo 2,6, O 2,9; longitud de la tegmina: holotipo 4,5; longitud del fémur posterior: holotipo 10,6; longitud de la tibia posterior: holotipo 9,8 .

DistRIBUCIÓN: provincia de Madrid.

Derivatio NOMINIS: dedicamos esta especie a la Dra. Vicenta Llorente, ortopteróloga del Museo Nacional de Ciencias Naturales de Madrid por confiarnos el estudio de los dos ejemplares de esta especie depositados en la colección de esta institución. 


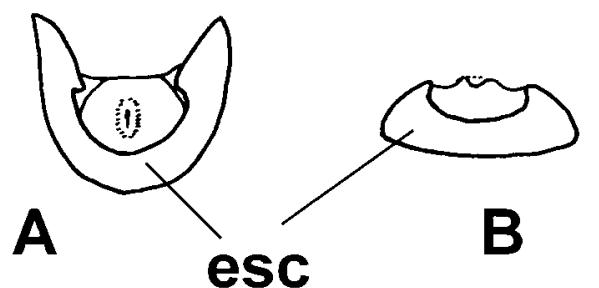

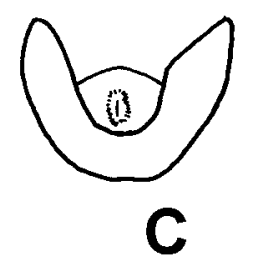
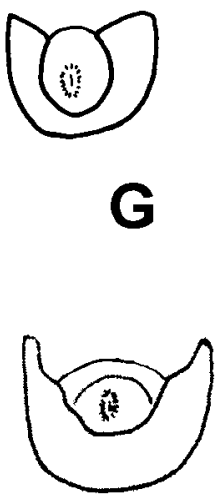

K
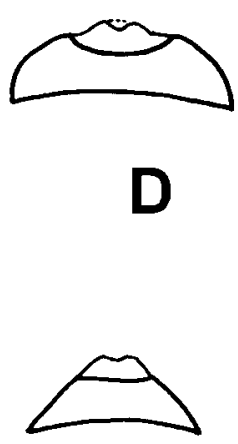

H

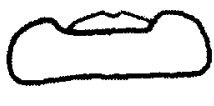

$\mathbf{L}$

Fig. 6.- Genitalia femenina, visión dorsal y anterior: A-B) P. mogon sp. n.; C-D) P. carabajali sp. n.; E-F) P. bolivari; G-H) $P$. barrancoi; I-J) P. venosa, K-L) P. baenai sp. n. Abreviaturas: esc) esclerito de la papila copulatriz.

Fig. 6.- Female Genitalia, dorsal and anterior views: A-B) P. mogon sp. n.; C-D) P. carabajali sp. n.; E-F) P. bolivari; G-H) P. barrancoi; I-J) P. venosa, K-L) P. baenai sp. n. Abreviations: esc) copulatory papilla esclerite.

Petaloptila (Zapetaloptila) bolivari (Cazurro, 1888)

(Figs. 2E; 2H; 3C; 4M-O; 5C; 6E-F)

La serie típica que sirvió para la descripción de esta especie se puede considerar extraviada, por lo que actualmente la única referencia es la descripción original de Cazurro (1888) y el trabajo de Pantel (1890) basado en ese material. Ambos artículos son ahora insuficientes para clarificar la posición de esta especie, pues se necesitan caracteres más finos y el estudio de la genitalia. En este trabajo se describen ejemplares de ambos sexos capturados dentro del área de distribución señalada en la descripción, recogiendo únicamente los caracteres no contemplados con anterioridad.

Material estudiado: $20^{7} \sigma^{7}$, Cova de les Rates Penades, Rótiva, Valencia, 1-IX-2002, 30SYJ367127, (A. Sendra et al., leg.); $20^{7} \sigma^{7}$, misma localidad, 19-I-2003; $30^{7} \sigma^{\pi}, 3$ 우 우, Cova de las Maravelles, Alzira, Valencia, 17-XI-2002, 30SYJ226339, trampa cerveza HC, (A. Sendra et al., leg.);
1 , Cova de las Ratetes, Corbera, Valencia, 17-XI-2002, 30SYJ2836, (A. Sendra et al., leg.). $30^{7} \sigma^{7}$ y 3 우 우 en la colección del autor, $10^{\pi}$ y 1 ㅇ depositados en M.N.C.N. de Madrid, el resto depositados en el Museo Valenciano de Historia Natural.

DESCRIPCIÓN DEL MACHO: el estudio de las glándulas dorsales y las tegminas, nos permiten confirmar que esta especie se incluye dentro del subgénero Zapetaloptila, como apuntaron Gorochov y Llorente (2001). Tegminas de coloración rojiza con el ápice y borde interno oscurecidos de negro, grandes (las más anchas de todas las especies del subgénero), adquiriendo un aspecto redondeado, donde se aprecian entre 4 y 5 venas bien marcadas (Fig. 2E). Glándula dorsal poco desarrollada en el segundo terguito abdominal, en el tercero aparece un repliegue en la mitad distal de modo que forma una protuberancia central junto al margen posterior (Fig. 2H). 
Epiprocto subcuadrangular, borde posterior levemente deprimido, casi recto; escotaduras laterales bien marcadas, amplia depresión central semicircular. En algunos ejemplares se observan areolas despigmentadas semicirculares alrededor de las escotaduras laterales y en el margen posterior (Fig. 3C). Lo que Cazurro (1888) denomina "Placa supraanal transversa, con dos prolongaciones en forma de cuernos", creemos que en realidad son los lóbulos pilosos del ápice del epifalo, pues en su posición natural, aparecen a partir del epiprocto y cubriendo parcialmente el borde de éste.

Lóbulos pilosos del ápice del epifalo cortos. Epifalo estrecho, desarticulado de los rami, con el borde anterior profundamente escotado en el medio, borde posterior muy prominente en el centro (Fig. 4M). Ectoparámero ancho, con la proyección distal no ramificada, borde superior de ésta muy engrosado formando una protuberancia alargada profusamente ornamentada de espínulas. Proyección proximal engrosada formando otra protuberancia alargada similar aunque más corta que la anterior, pero igualmente ornamentada (Fig. $4 \mathrm{~N}$ O). Apodemas endoparamerales anchos, más cortos que los rami. Moldura de la placa de sujeción del espermatóforo muy ancha, escotada en el borde anterior con expansiones laterales grandes, borde posterior convexo en el centro y con dos grandes procesos espiniformes separados un tercio de la anchura de la moldura. Arco del endoparámero muy ancho y grueso, cóndilos no marcados. Varilla guía bilobulada en el ápice.

DESCRIPCIÓN DE LA HEMBRA: placa subgenital ampliamente escotada en el borde posterior. Epiprocto subtrapezoidal con el margen posterior cóncavo y muy piloso; márgenes laterales poco escotados; mitad anterior de la placa supranal con una depresión triangular central (Fig. 5C), en general muy parecido a P. mogon. Esclerito de la papila copulatriz anular en visión dorsal, levemente aplanada porteriormente con un pequeño hueco central (Fig. 6E); trapezoidal en visión anterior con una escotadura inferior central (Fig. 6F).

DisTRIBUCIÓN: en las cuevas de las provincias de Valencia y Alicante.

Medidas (en milímetros): longitud total: $\sigma^{\top} 15,7-17,9$, o 15,3-21,9; longitud pronoto: $\sigma^{\top} 2,8$ 2,9 , ㅇ 2,9-3,2; longitud de la tegmina: $\sigma^{7} 4,3-5,3$; anchura de la tegmina: $\sigma^{7} 2,2-3,1$; longitud del fémur posterior: O' $11-11,9$, $\$ 11,5-14,1$; longitud de la tibia posterior: $0^{7} 9,6-10,4$, $\$ 9,4-11,7$; longitud del oviscapto: $12,5-15,7$.
Petaloptila (Zapetaloptila) barrancoi Gorochov \& Llorente, 2001

(Figs. 3D; 5D; 6G-H)

Material estudiado: $30^{7} \sigma^{7}, 3$ 우 $ᄋ$, cueva del Lobo, t. m. Enix, Sierra de Gádor, Almería, 12-V-2001, (P. Barranco leg.).

MACHO: Epiprocto subtrapezoidal, margen posterior recto, escotaduras laterales muy poco marcadas, tercio anterior con una amplia depresión triangular (Fig. 3D).

HemBRA: Epiprocto grande, cubierto de profusa pilosidad, prolongado posteriormente y convexo, márgenes laterales expandidos sin escotaduras laterales (Fig. 5D).

Placa subgenital lisa, muy levemente escotada en el borde posterior. Papila copulatriz en visión dorsal casi anular, abierta posteriormente, con los extremos de las ramas truncados (Fig. 6G). Esclerito cónico en visión anterior (Fig. 6H).

MEDIDAS (en milímetros): longitud total: $\sigma^{7} 14,8-16,9$, 우 17,6-20,5; longitud pronoto: $\sigma^{7} 2,4-$ 2,7, ㅇ 3-3,3; longitud de la tegmina: $\sigma^{7} 5,6-5,8$; longitud del fémur posterior: $\sigma^{7} 11,4-12$, ㅇ 13-14,5; longitud de la tibia posterior: ơ 10-10,9, 우 11,112,1; longitud del oviscapto: 12-13,3.

DistribuCión: cuevas de las provincias de Almería y Granada.

Petaloptila (Zapetaloptila) venosa Gorochov \& Llorente, 2001

(Figs. 3E; 5E; 6I-J)

Material estudiado: Holotipo: $1 \sigma^{7}$, Teruel, $935 \mathrm{~m}, 10$-VIII-33, (B. Muñoz), M.N.C.N. Cat. Tipos $\mathrm{N}^{\circ} 8828 ; 30^{x} 0^{x}, 9$ 우 우, Cova de l'Onder, Aín, Castellón, 14-XII-2002, 30SYK269203, trampa de cerveza-HC y trampas cebo, (J. Albesa et al. leg.); 3 우 우, Cova Oscura, Atzaneta, Castellón, 30TYK371538, (J. Albesa leg.); 1 오, Avenc d'en Serenge, Cabanes, Castellón, 2-II-2003, (S. Montagud et al. leg.). $20^{7} 0^{7}$ y 3 우 우 en la colección del autor, $10^{7}$ y 2 우 우 depositados en M.N.C.N. de Madrid, el resto depositados en el Museo Valenciano de Historia Natural.

Se describe aquí la hembra de esta especie por ser hasta el momento desconocida y se aportan nuevos aspectos a la descripción del macho.

МАсно: epiprocto subtrapezoidal, margen posterior cóncavo, bastante más deprimido que en las otras especies, escotaduras marginales poco marcadas, mitad anterior con una pequeña depresión central semicircular (Fig. 3E).

DESCRIPCIÓN DE LA HEMBRA: Coloración general semejante a la del macho indicada en Gorochov y Llorente (2001). Tibias posteriores armadas con 4 
Tabla 1.- Valores medios en milímetros de varios parámetros para las especies del subgénero Zapetaloptila. F: longitud del fémur posterior, T: longitud de la tibia posterior, TG: longitud de la tegmina, L/A: relación entre la longitud y anchura de la tegmina, PR: longitud del pronoto, LT: longitud total, O: longitud del oviscapto. $\left(^{*}\right)$ Medidas tomadas de ejemplares conservados en seco.

Table 1.- Mean values in milimeters of the measurements of the subgenus Zapetaloptila species. F: length of hind femur, T: length of hind tibia, TG: lenght of tegmen, L/A: tegmen lenght/wide ratio, PR: lenght of pronotum, LT: lenght of body, O: lenght of ovipositor. $(*)$ Measurements from dry preserved material.

\begin{tabular}{lcccccccc}
\hline & \multicolumn{1}{c}{} & \multicolumn{1}{c}{$\sigma^{\pi} \sigma^{\pi}$} \\
\hline & $\mathrm{n}$ & $\mathrm{F}$ & $\mathrm{T}$ & $\mathrm{TG}$ & $\mathrm{L} / \mathrm{A}$ & $\mathrm{PR}$ & $\mathrm{LT}$ & LT/TG \\
\hline P. mogon sp. n. & 4 & 13,4 & 11,5 & 5,8 & 2 & 3 & 19,6 & 3,38 \\
P. baenai sp. n. & 6 & 9,8 & 8,1 & 4,3 & 2,02 & 2,4 & 14,9 & 3,43 \\
P. carabajali sp. n. & 1 & 11,2 & 10,3 & 5,4 & 2,14 & 2,9 & 14,8 & 2,74 \\
P. llorenteae sp. n. & 1 & 10,6 & 9,8 & 4,5 & 2,21 & 2,6 & 12,4 & 2,76 \\
P. barrancoi & 3 & 12,1 & 10,5 & 5,7 & 2,3 & 2,6 & 15,7 & 2,75 \\
P. venosa & 3 & 13,3 & 11,3 & 6,2 & 2,20 & 2,5 & 19,7 & 3,18 \\
P. bolivari & 4 & 11,6 & 10,1 & 5,1 & 1,81 & 2,9 & 16,9 & 3,31 \\
\hline
\end{tabular}

\begin{tabular}{lccccccc}
\hline & \multicolumn{7}{c}{ \% 9} \\
\hline & $\mathrm{n}$ & $\mathrm{F}$ & $\mathrm{T}$ & $\mathrm{PR}$ & $\mathrm{LT}$ & $\mathrm{O}$ & $\mathrm{F} / \mathrm{O}$ \\
\hline P. mogon sp. n. & 4 & 13,8 & 11,7 & 3,2 & 22,5 & 15 & 0,92 \\
P. baenai sp. n. & 1 & 11,4 & 9,2 & 2,8 & 18,1 & 12,5 & 0,91 \\
P. carabajali sp. n. & 2 & 11,6 & 11,7 & 2,7 & 16,9 & 12,3 & 0,94 \\
P. llorenteae sp. n. & 1 & - & - & 2,9 & 13,6 & - & - \\
P. barrancoi & 3 & 13,8 & 11,6 & 3,1 & 19,5 & 12,7 & 1,09 \\
P. venosa & 6 & 11,6 & 9,6 & 3,1 & 13,7 & 12,7 & 0,91 \\
$P$. bolivari & 4 & 12,7 & 10,7 & 3,1 & 19,5 & 14,0 & 0,91 \\
\hline
\end{tabular}

espolones tanto externos como internos y de 8-10 espinas internas y 10-12 externas. Epiprocto subtrapezoidal, corto, con el borde posterior casi recto, escotaduras laterales poco marcadas, mitad anterior con una pequeña depresión subcircular central (Fig. 5E). Placa subgenital subtriangular y levemente escotada como en P. bolivari. Oviscapto castaño, curvado hacia abajo, tanto o más largo que la longitud del fémur posterior. Papila copulatriz acorazonada, esclerito totalmente cerrado con una abertura hexagonal y central amplia en cuyo centro se sitúa el orificio del conducto de la espermateca, sinuoso posteriormente (Fig. 6I). Esclerito escotado en el margen inferior en visión anterior (Fig. 6J).

MEDIDAS (en milímetros): longitud total: $\sigma^{\top} 19,1-20,3$, ㅇ 16,3-19,3; longitud pronoto: $0^{\top} 2,5-$ 2,6 , ㅇ 2,8-4,1; longitud de la tegmina: $0^{7} 4,7-6,9$; anchura de la tegmina: $\sigma^{7} 2,2-3,1$; longitud del fémur posterior: $\sigma^{\pi} 13,1-13,3$, \& 11,2-12,6; longitud de la tibia posterior: $0^{\pi} 11-11,6$, $ᄋ$ 9,1-10,9; longitud del oviscapto: 11,5-14,9.
DistRIBUCIÓn: cuevas de las provincias de Teruel y Castellón.

\section{Discusión}

Los machos de mayor tamaño son los de $P$. mogon y $P$. venosa, y las hembras de mayor tamaño son $P$. mogon, seguidas de $P$. barrancoi y $P$. bolivari. Por lo que se puede considerar a $P$. mogon como la especie de mayor tamaño del género, incluida $P$. andreini según los valores recogidos en Harz (1969), Olmo-Vidal y Hernando (2000) y Gorochov y Llorente (2001). Por lo general las especies del subgénero Petaloptila son de menor tamaño que las del subgénero Zapetaloptila, y dentro del cual las más pequeñas son $P$. llorenteae y $P$. carabajali, si bien las medidas de la primera están realizadas sobre ejemplares en seco (Tabla 1). Por otro lado de $P$. venosa se han medido mayor número de hembras y sorprendentemente la hembra es de menor tamaño que el macho. 
En cuanto a la venación de las tegminas, creemos que existe una evaluación subjetiva de su manifestación, puesto que si se observan con detenimiento las tegminas de las especies hasta ahora conocidas del subgénero Zapetaloptila, se constata que no se corresponden con lo que se recoge en el trabajo de Gorochov y Llorente (2001), puesto que ni $P$. barrancoi tiene ausencia total de venación, pueden apreciarse hasta 4 venas al trasluz, ni $P$. venosa posee tantas como se refleja en la figura original. En esta especie, después de estudiar el holotipo y varios ejemplares de Castellón, podemos señalar que el número de venas es variable de 4 a 6 , si bien la profusión se debe a la bifurcación de algunas de ellas. Por otro lado es interesante destacar la diferente forma que presentan las tegminas en cada especie y su mayor o menor desarrollo; lo primero viene indicado por la relación longitud-anchura de la tegmina y lo segundo por la relación longitud total y longitud de la tegmina. De este modo, la tegmina más ancha aparece en $P$. bolivari con un valor tan sólo de 1,81 frente a 2,3 de $P$. barrancoi que es la más estrecha. $P$. venosa es la que presenta unas tegminas de mayor tamaño, seguida de $P$. mogon, siendo las más cortas las de P. llorenteae y P. baenai (Tabla 1). Si bien en proporción al tamaño del cuerpo, hay tres especies que presentan un valor similar $P$. carabajali, $P$. llorenteae y $P$. barrancoi. En estas tres especies la tegmina alcanza el borde posterior del tercer segmento abdominal. En este sentido, las tegminas que menos cubren el dorso del abdomen son las de P. baenai y P. mogon.

Un carácter que se indica siempre en la descripción de las hembras es la relación entre la longitud del fémur posterior y el oviscapto ( $\mathrm{F} / \mathrm{O}$ en la Tabla 1), P. barrancoi es la única especie en la que este valor es superior a la unidad, pues su oviscapto es más corto que el fémur posterior. Para el resto de las especies este valor es muy similar.

Las cuatro nuevas especies descritas parecen pertenecer a un tronco común en relación a la genitalia masculina. Todas comparten una proyección distal del ectoparámero de aspecto bivalvo, aunque los bordes internos y externos de éste y la forma de la proyección proximal es diferente en cada caso. P. baenai se separa claramente de las otras tres porque la proyección proximal del ectoparámero es estrecha y prolongada, mientras que en las otras tres es corta y ancha, siendo particularmente curvada en $P$. mogon. En relación a la genitalia femenina también existe relación entre $P$. mogon, $P$. baenai y $P$. carabajali, pues estas tres especies comparten el esclerito de la papila copu- latriz en forma de herradura. Por otro lado, $P$. barrancoi y $P$. bolivari presentan el esclerito de la papila copulatriz en forma de anillo incompleto, si bien es más pequeño en la primera y muy voluminoso en la segunda.

Claves de identificación de las especies del subgénero Zapetaloptila:

\section{A) MACHOS:}

1. Ectoparámeros con la proyección distal no ramificada .... 2

1'. Ectoparámeros con la proyección distal ramificada ......... 3

2. Ectoparámero de aspecto bivalvo (Figs. 4B, E, H, K) .... 4

2'. Ectoparámero sin aspecto bivalvo, proyecciones engrosadas y ornamentadas con densas espínulas (Fig. 4N) ........ P. bolivari (Cazurro, 1888)

3. Ramificación posterior del ectoparámero grande y dirigida hacia abajo, ramificación anterior pequeña y dirigida hacia abajo (Figs. 5G, H en Gorochov y Llorente, 2001)

P. venosa Gorochov \& Llorente, 2001

3'. Ramificación posterior del ectoparámero pequeña y dirigida ligeramente hacia abajo y atrás, ramificación anterior grande y dirigida hacia delante (Figs. 5J, K en Gorochov \& Llorente, 2001)

P. barrancoi Gorochov \& Llorente, 2001

4. Tegminas superan el borde posterior del tercer terguito abdominal P. carabajali sp. n.

4'. Tegminas no alcanzan el borde posterior del tercer terguito abdominal ................................................................ 5

5. Escotaduras laterales del epiprocto bien marcadas ........... P. $\operatorname{mogon}$ sp. n.

5'. Escotaduras laterales del epiprocto poco marcadas ...... 6

6. Proyección proximal del ectoparámero estrecha y prolongada en un tubérculo (Fig. 4E) ............... P. baenai sp. n.

6'. Proyección proximal del ectoparámero corta y roma (Fig. $4 \mathrm{~K})$............................................... Plorenteae sp. $\mathrm{n}$.

B) Hembras:

1. Coloración general dorsal pálida ................................... 2

1'. Coloración general dorsal castaño oscuro ......................

2. Oviscapto más corto que el fémur posterior P. barrancoi Gorochov \& Llorente, 2001.

2'. Oviscapto igual o más largo que el fémur posterior ....... 3

3. Esclerito de la papila copulatriz abierto anteriormente .... 4

3'. Esclerito de la papila copulatriz cerrado anteriormente, acorazonado (Fig. 6I)

P. venosa Gorochov \& Llorente, 2001.

4. Epiprocto grande con los márgenes laterales y posterior expandidos ........................................ P. llorenteae sp. n.

4'. Epiprocto corto, transverso con los márgenes laterales no expandidos ..................................................................... 5

5. Esclerito de la papila copulatriz con forma de herradura .. 6

5'. Esclerito de la papila copulatriz con forma anular (Fig. 6E) P. bolivari (Cazurro, 1888).

6. Bordes laterales de la papila copulatriz divergentes (Fig.

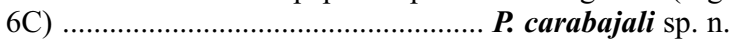

6'. Bordes laterales de la papila copulatriz paralelos (Fig. 6K) P. baenai sp. n. 


\section{AGRADECIMIENTOS}

Queremos expresar nuestro agradecimiento a todas aquellas personas que han contribuido a la captura de los ejemplares que han servido para la realización del presente estudio y a las que nos los han enviado. Especialmente a los componentes del Grupo Espeleológico de Villacarrillo (GEV) de Jaén que han capturado el material que ha servido para la descripción de P. mogon sp. nov. A don Manuel Baena que nos ha enviado dicho material junto con otro de Córdoba y Jaén que ha servido para la descripción del P. baenai sp. nov. A don Emilio Carabajal que nos ha cedido el material de la Sierra de Cazorla y que ha servido para la descripción de P. carabajali sp. nov. A la Dra. Vicenta Llorente del M.N.C.N. que nos ha confiado los dos ejemplares de Rivas Vaciamadrid y que han servido para la descripción de P. llorenteae sp. nov. Y al Dr. Alberto Sendra por enviarnos el material del Levante peninsular de las especies $P$. venosa y $P$. bolivari.

\section{Referencias}

Alexander, R. D. \& OTte, D., 1967. The evolution of genitalia and mating behaviour in crickets (Gryllidae) and other Orthoptera. Miscellaneous Publications. Museum of Zoology, University of Michigan, no. 133, 62 pp.

Barranco, P., BAena, M. Y PAscual, F., 1996. Los Orthoptera de la provincia de Córdoba. Zoologica baetica, 7:61-77.

Cazurro, M., 1888. Ortópteros de España y Portugal. Anales de la Sociedad Española de Historia Natural, 17: 457-465.

Gorochov, A. V. \& Llorente, V., 2001. Estudio taxonómico preliminar de los Grylloidea de España (Insecta, Orthoptera). Graellsia, 57: 95-139.
Hubbell, T. H. \& Norton, R. M., 1978. The systematics and biology of the cave-crickets of the North American tribe Hadenoecini (Orthoptera Saltatoria: Ensifera: Rhaphidophoridae: Dolichopodinae). Miscellaneous Publications, Museum of Zoology, University of Michigan, $\mathrm{N}^{\mathrm{o}} 156,124 \mathrm{pp} .+\mathrm{V}$ plates.

Mesa, A., García, P. C. \& ZeFA, E., 1996. Strinatia brevipennis Chopard 1970 and S. teresopolis sp. n.: description of new species and comparative study of their chromosomes and male and female genitalia sclerites (Grylloidea, Phalangosidae). Journal of Orthoptera Research, 8: 73-82.

Olmo-Vidal, J. M. \& Hernando, C., 2000. A new cave cricket of the genus Petaloptila from the Pyrenean mountains (N. Iberian peninsula) (Orthoptera: Gryllidae). Journal of Orthoptera Research, 9: 135-138.

OtTe, D. \& AleXANDER, R.D., 1983. The Australian crickets (Orthoptera: Gryllidae). Academy of Natural Sciences of Philadelphia. Philadelphia. 477 pp.

PANTEL, P.J., 1890. Notes orthoptèrologiques. I. Révision monographique du genre Gryllomorpha Fieb. Anales de la Sociedad Española de Historia Natural, 19: 335-370. 